\title{
A short proof of the Gaillard-Matveev theorem based on shape invariance arguments
}

\author{
Y. Grandati \\ Equipe BioPhyStat, LCP A2MC, ICPMB, IF CNRS 2843, \\ Université de Lorraine-Site de Metz, 1 Bd Arago, 57078 Metz, Cedex 3, France
}

\begin{abstract}
We propose a simple alternative proof of the Wronskian representation formula obtained by Gaillard and Matveev for the Darboux-Pöschl-Teller (DPT) potentials. It rests on the use of singular Darboux-Bäcklund transformations applied to the free particle system combined to the shape invariance properties of the DPT.
\end{abstract}

PACS numbers:

\section{INTRODUCTION}

In 2002, Gaillard and Matveev established an interesting connection between the Darboux-Crum dressing formula and the Darboux-Pöschl-Teller potentials. More precisely, they proved the following theorem:

Gaillard-Matveev's theorem: The trigonometric Darboux-Pöschl-Teller potential (TDPT) with integer parameters $m \geq n$ defined on $x \in] 0, \pi / 2[$ by

$$
V(x ; m, n)=\frac{m(m+1)}{\sin ^{2} x}+\frac{n(n+1)}{\cos ^{2} x},
$$

admits the following Wronskian representation

$$
V(x ; m, n)=-2\left(\ln W\left(u_{1}, \ldots, u_{m} \mid x\right)\right)^{\prime \prime},
$$

where $W\left(u_{1}, \ldots, u_{m} \mid x\right)$ denotes the Wronskian of the functions $u_{1}(x), \ldots, u_{m}(x)$ which are given by

$$
\left\{\begin{array}{c}
u_{k}(x)=\sin (l x), \text { if } 1 \leq l \leq m-n \\
u_{m-n+l}(x)=\sin ((m-n+2 l) x), \text { if } 1 \leq l \leq n .
\end{array}\right.
$$

Gaillard and Matveev underlined that this result traduces the existence of a chain of Darboux transformations connecting the constant potential to the TDPT $V(x ; m, n)$. If such a construction was already envisaged in Darboux's seminal works $\frac{1}{\text {, }}$, the theorem above precises what is the correct choice of "seeds" eigenfunctions of the free Hamiltonian to reach this last potential.

The proof given $\mathrm{in}^{2}$ and ${ }^{3}$ rests on direct evaluations of the Wronskian $W\left(u_{1}, \ldots, u_{m} \mid x\right)$ and is quite sophisticated and technically involved. In this letter, we show that this result can be obtained in a shorter way directly by following Darboux's original idea. In a modern formulation the proof makes use of shape invariance and SUSY QM arguments associated to singular Darboux-Bäcklund Transformations (DBT) built from excited eigenstates. If the use of singular DBT has already been envisaged in several papers ${ }^{4-7}$, they are generally considered of less interest, although recently proven to be crucial in the study of some two-dimensional superintegrable systems $\stackrel{8}{-}$.

We also examine the case of the Bessel potentials, which is the first example envisaged by Darboux ${ }^{1}$. This is a confluent case which necessitate to employ Matveev's generalized Wronskian leading then to a Wronskian version of the Rayleigh formula for Bessel functions.

\section{DARBOUX-BÄCKLUND TRANSFORMATIONS}

We begin to briefly recall ther essential features of DBT and shape invariance. If $\psi_{\lambda}(x ; a)$ is an eigenstate of the hamiltonian $\widehat{H}(a)=-d^{2} / d x^{2}+V(x ; a), x \in I \subset \mathbb{R},\left(a \in \mathbb{R}^{m}\right.$ being a multiparameter $)$ associated to the eigenvalue $E_{\lambda}(a)\left(E_{0}(a)=0\right)$ 


$$
\psi_{\lambda}^{\prime \prime}(x ; a)+\left(E_{\lambda}(a)-V(x ; a)\right) \psi_{\lambda}(x ; a)=0,
$$

then the Riccati-Schrödinger (RS) function $w_{\lambda}(x ; a)=-\psi_{\lambda}^{\prime}(x ; a) / \psi_{\lambda}(x ; a)$ satisfies the corresponding RiccatiSchrödinger (RS) equation ${ }^{9}$

$$
-w_{\lambda}^{\prime}(x ; a)+w_{\lambda}^{2}(x ; a)=V(x ; a)-E_{\lambda}(a) .
$$

The class of RS equations is preserved by a specific subset of the group $\mathcal{G}$ of smooth $S L(2, \mathbb{R})$-valued curves $\operatorname{Map}(\mathbb{R}, S L(2, \mathbb{R}))$. These transformations, called Darboux-Bäcklund Transformations (DBT), are built from any solution $w_{\nu}(x ; a)$ of the initial RS equation $\mathrm{Eq}(\underline{5})$ as ${ }^{\underline{9}}-11$

$$
w_{\lambda}(x ; a) \stackrel{A\left(w_{\nu}\right)}{\rightarrow} w_{\lambda}^{(\nu)}(x ; a)=-w_{\nu}(x ; a)+\frac{E_{\lambda}(a)-E_{\nu}(a)}{w_{\nu}(x ; a)-w_{\lambda}(x ; a)},
$$

where $E_{\lambda}(a) \neq E_{\nu}(a) \cdot w_{\lambda}^{(\nu)}$ is then a solution of the RS equation:

$$
-w_{\lambda}^{(\nu) \prime}(x ; a)+\left(w_{\lambda}^{(\nu)}(x ; a)\right)^{2}=V^{(\nu)}(x ; a)-E_{\lambda}(a),
$$

with the same energy $E_{\lambda}(a)$ as in Eq(5) but with a modified potential

$$
V^{(\nu)}(x ; a)=V(x ; a)+2 w_{\nu}^{\prime}(x ; a)
$$

called an extension of $V$. If $V^{(\nu)}$ is a rational function of an a priori given variable $(x, \tan x, \sin x, \cosh x \ldots)$ we call it a rational extension of $V$.

This can be schematically summarized as

$$
\left\{\begin{array}{l}
w_{\lambda} \stackrel{A\left(w_{\nu}\right)}{\longmapsto} w_{\lambda}^{(\nu)} \\
V \stackrel{A\left(w_{\nu}\right)}{\longmapsto} V^{(\nu)} .
\end{array}\right.
$$

The corresponding eigenstate of $\widehat{H}^{(\nu)}(a)=-d^{2} / d x^{2}+V^{(\nu)}(x ; a)$ can be written

$$
\psi_{\lambda}^{(\nu)}(x ; a)=\exp \left(-\int d x w_{\lambda}^{(\nu)}(x ; a)\right) \sim \frac{1}{\sqrt{E_{\lambda}(a)-E_{\nu}(a)}} \widehat{A}\left(w_{\nu}\right) \psi_{\lambda}(x ; a)
$$

where $\widehat{A}\left(w_{\nu}\right)$ is a first order operator given by $\widehat{A}\left(w_{\nu}\right)=d / d x+w_{\nu}(x ; a)$. Eq(10) can still be written as

$$
\psi_{\lambda}^{(\nu)}(x ; a) \sim \frac{W\left(\psi_{\nu}, \psi_{\lambda} \mid x\right)}{\psi_{\nu}(x ; a)},
$$

where $W\left(y_{1}, \ldots, y_{n} \mid x\right)$ is the Wronskian of the functions $y_{1}, \ldots, y_{n}$.

From $V$, the DBT generates a new potential $V^{(\nu)}$ (quasi) isospectral to the original one and its eigenfunctions are directly obtained from those of $V$ via $\mathrm{Eq}(10)$. Nevertheless, in general, $w_{\nu}(x ; a)$ and then the transformed potential $V^{(\nu)}(x ; a)$ is singular at the nodes of $\psi_{\nu}(x ; a)$. For instance, if $\widehat{H}(a)$ admits a bound state spectrum $\left(E_{n}, \psi_{n}\right)_{n \in \mathbb{N}}$ $(x ; a), V^{(n)}$ is regular only when $n=0$, that is when $\psi_{n=0}$ is the ground state of $\widehat{H}$, and we recover the usual SUSY partnership in quantum mechanics 17,18 . Note that $A\left(w_{0}\right)$ is a "state-deleting" DBT, the spectrum of the superpartner hamiltonian $\widehat{\widetilde{H}}=\widehat{H}^{(0)}$ having for fundamental level and ground state $E_{1}$ and $\psi_{1}^{(0)}$ respectively.

$V$ is said to be a translationally shape invariant potential (TSIP) if its superpartner has the form

$$
\widetilde{V}(x ; a)=V^{(0)}(x ; a)=V(x ; a+\alpha)+R(a),
$$


where $\alpha \in \mathbb{R}^{m}$.

The question of successive iterations of DBT is very natural and is at the center of the construction of the hierarchy of hamiltonians in the usual SUSY QM scheme ${ }^{12}$. Staying at the formal level, it can be simply described by the following generalization of $\operatorname{Eq}(9)\left(N_{j}\right.$ denotes the j-uple $\left(\nu_{1},, \ldots, \nu_{j}\right)$, with $N_{1}=\nu_{1}$ and $\left.N_{m}=\left(N_{m-1}, \nu_{m}\right)\right)$

$$
\left\{\begin{array}{c}
w_{\lambda} \stackrel{A\left(w_{\nu_{1}}\right)}{\longmapsto} w_{\lambda}^{\left(\nu_{1}\right)} \stackrel{A\left(w_{\nu_{2}}^{\left(N_{1}\right)}\right)}{\longmapsto} w_{\lambda}^{\left(N_{2}\right)} \ldots \stackrel{A\left(w_{\nu_{m}}^{\left(N_{m}-1\right)}\right)}{\longmapsto} w_{\lambda}^{\left(N_{m}\right)} \\
V \stackrel{A\left(w_{\nu_{1}}\right)}{\longmapsto} V^{\left(\nu_{1}\right)} \stackrel{A(\underbrace{\left(N_{1}\right)}_{\nu_{2}})}{\longmapsto} V^{\left(N_{2}\right)} \ldots \stackrel{A\left(w_{\nu_{m}}^{\left(N_{m}\right)}\right)}{\longmapsto} V^{\left(N_{m}\right)},
\end{array}\right.
$$

where $w_{\lambda}^{\left(N_{m}\right)}$ is a RS function associated to the eigenvalue $E_{\lambda}$ of the potential

$$
V^{\left(N_{m}\right)}(x ; a)=V(x ; a)+2 \sum_{j=1}^{m}\left(w_{\nu_{j}}^{\left(N_{j-1}\right)}(x ; a)\right)^{\prime}
$$

The corresponding eigenfunction is given by (cf Eq(10) and Eq(11) )

$$
\psi_{\lambda}^{\left(N_{m}\right)}(x ; a)=\widehat{A}\left(w_{\nu_{m}}^{\left(N_{m-1}\right)}\right) \psi_{\lambda}^{\left(N_{m-1}\right)}(x ; a)=\frac{W\left(\psi_{\nu_{m}}^{\left(N_{m-1}\right)}, \psi_{\lambda}^{\left(N_{m-1}\right)} \mid x\right)}{\psi_{\nu_{m}}^{\left(N_{m-1}\right)}(x ; a)}
$$

or

$$
\psi_{\lambda}^{\left(N_{m}\right)}(x ; a)=\widehat{A}\left(w_{\nu_{m}}^{\left(N_{m-1}\right)}\right) \ldots \widehat{A}\left(w_{\nu_{1}}\right) \psi_{\lambda}(x ; a)
$$

By induction, we can prove the Crum formulas 13,14

$$
\left\{\begin{array}{c}
\psi_{\lambda}^{\left(N_{m}\right)}(x ; a)=W\left(\psi_{\nu_{1}}, \ldots, \psi_{\nu_{m}}, \psi_{\lambda} \mid x\right) / W\left(\psi_{\nu_{1}}, \ldots, \psi_{\nu_{m}} \mid x\right) \\
V^{\left(N_{m}\right)}(x ; a)=V(x ; a)-2\left(\log W\left(\psi_{\nu_{1}}, \ldots, \psi_{\nu_{m}} \mid x\right)\right)^{\prime \prime}
\end{array}\right.
$$

A succession of $m$ SUSY QM partnerships gives rise to a hierarchy of regular potentials $V^{\left(N_{j}\right)}, j=1, \ldots, m$, associated to the m-uple $N_{m}=(0,, \ldots, m-1)$, that is, to a set of seeds functions $\left(\psi_{0}, \ldots, \psi_{m-1}\right)$ constituted by the $m$ first bound states of the initial hamiltonian. In the case where the initial potential is a TSIP, we obtain simply

$$
V^{\left(N_{m}\right)}(x ; a)=V(x ; a)-2\left(\log W\left(\psi_{0}, \ldots, \psi_{m-1} \mid x\right)\right)^{\prime \prime}=V(x ; a+m \alpha)+\sum_{j=0}^{m-1} R\left(a_{j}\right)
$$

with

$$
\psi_{k+m}^{\left(N_{m}\right)}(x ; a)=\frac{W\left(\psi_{0}, \ldots, \psi_{m-1}, \psi_{k+m} \mid x\right)}{W\left(\psi_{0}, \ldots, \psi_{m-1} \mid x\right)}, \quad k \geq 0
$$

which is the $k$-th excited state of $V^{\left(N_{m}\right)}$ with the associated energy $E_{k+m}$.

\section{FROM THE CONSTANT POTENTIAL TO THE TDPT POTENTIAL}

Following Darboux ${ }^{1}$, our starting point is the constant potential on the real line $V(x)=0, x \in \mathbb{R}$. The physical energy spectrum of the corresponding hamiltonian $\widehat{H}$ is constituted by an open band $\left.\mathcal{E}_{k}=k^{2} \in\right] 0,+\infty[, k>0$, where the two-dimensional eigenspace associated to $\mathcal{E}_{k}$ is spanned by the (unnormalized) scattering eigenstates

$$
\phi_{k}^{\text {odd }}(x)=\sin (k x) / k, \phi_{k}^{\text {even }}(x)=\cos (k x) / k \text {. }
$$


We then build a chain of extensions of $V$ where the first DBT we choose is based on a scattering state satisfying the Dirichlet boundary condition at the origin, namely

$$
\psi_{0}(x)=\phi_{1}^{\text {odd }}(x)=\sin (x), w_{0}(x)=-\cot (x),
$$

where the choice of the value $k=1$ is for pure convenience. The first extended potential is then

$$
V^{(0)}(x)=V(x)+2 w_{0}^{\prime}(x)=\frac{2}{\sin ^{2} x}
$$

and the corresponding Schrödinger equation

$$
\left(-d^{2} / d x^{2}+V^{(0)}(x)\right) \psi(x)=E \psi(x)
$$

At this step, Darboux ${ }^{1}$ writes

"Pour des valeurs particulières de E, cette équation admet les solutions $\sin ^{2} x, \sin ^{2} x \cos x$. En les employant et en poursuivant l'application de la méthode, on parviendra successivement à des équations de la forme

$$
\left(-d^{2} / d x^{2}+\frac{n(n+1)}{\cos ^{2} x}+\frac{m(m+1)}{\sin ^{2} x}\right) \psi(x)=E \psi(x) . "
$$

As emphasized by Gaillard and Matveev, the argument needs to be completed and specified in particular to determine in a systematic way what is the correct choice of the successive seeds functions to reach this equation.

First note that Darboux's procedure does not consider the spectral properties of the built hamiltonians which can then become more singular at each step. This is the case of $V^{(0)}$ which is singular at every node $\xi_{l}=l \pi$ of $\psi_{0}(x)$. The corresponding singularities are of the centrifugal barrier $\lambda /\left(x-\xi_{l}\right)^{2}$ with $\lambda>3 / 4$, that is, strong singularities for which the transmission probability is zero $5,15,16$. This confines the particle in a single interval $] \xi_{l}, \xi_{l+1}[$. If we take for instance $l=0$, the existence of the singularities in 0 and $\pi$ can then be considered as equivalent to the addition of Dirichlet boundary conditions at the origin and at $x=\pi$.

$$
\psi\left(0^{+}\right)=\psi\left(\pi^{-}\right)=0
$$

These lasts act as a filter which select among all the formal eigenfunctions $\psi_{k}^{(0)}$ only those which satisfy Eq(24). The question is now to determine what are the appropriate values of $k$ which allows to verify this constraint.

From a more general point of view, consider the problem to determine what is the condition to which the solution $\psi_{\lambda}(x)$ of Eq(4) is submitted in order that its image $\psi_{\lambda}^{(\nu)}$ via the $\operatorname{DBT} A\left(w_{\nu}\right)$ satifies

$$
\psi_{\lambda}\left(x_{0}\right)=0
$$

where $x_{0} \in I$ is a zero of $\psi_{\nu}$. In the vinicity of $x_{0}$ we can write

$$
\psi_{\mu}(x)=a_{\mu, 0}+a_{\mu, 1}\left(x-x_{0}\right)+a_{\mu, 2}\left(x-x_{0}\right)^{2}+O\left(\left(x-x_{0}\right)^{3}\right) .
$$

$x_{0}$ being a simple zero of $\psi_{\nu}$ we have $a_{\nu, 0}=0$ and $a_{\nu, 1} \neq 0$, which gives

$$
w_{\nu}(x) \underset{x \rightarrow x_{0}}{\simeq} \frac{-1}{x-x_{0}}-\frac{a_{\nu, 2}}{a_{\nu, 1}}+O\left(\left(x-x_{0}\right)\right) .
$$

Inserting these results in Eq(11), we obtain

$$
\psi_{\lambda}^{(\nu)}(x) \underset{x \rightarrow x_{0}}{\sim} \frac{-a_{\lambda, 0}}{x-x_{0}}-\frac{a_{\nu, 2}}{a_{\nu, 1}} a_{\lambda, 0}+O\left(\left(x-x_{0}\right)\right)
$$

and the condition (25) is satisfied iff $a_{\lambda, 0}=0$, that is, iff $x_{0}$ is also a zero of $\psi_{\lambda}$. 
In brief, a DBT $A\left(w_{\nu}\right)$ associated to a seed function $\psi_{\nu}$ having nodes on the definition interval generates an extended hamiltonian which is singular on this interval. The restriction of this extension between two singularities has a spectrum which consists in a filtered version of the initial hamiltonian spectrum: we only keep the levels $E_{\lambda}$ for which the initial eigenstates $\psi_{\lambda}$ have nodes at the singular points. This point of view is equivalent to the one of Marquez et al $\stackrel{7}{ }$.

Applying this analysis to to the system considered above, we obtain that the potential $V^{(0)}$ restricted to the positive half line and subjected to the boundary conditions Eq(24) has a discrete non degenerate spectrum given by $E_{l}, l \in \mathbb{N}^{*}$, with the corresponding eigenstates

$$
\psi_{l}^{(0)}(x)=\widehat{A}\left(w_{0}\right) \psi_{l}(x) \sim(l+1) \cos ((l+1) x)-\cot x \sin ((l+1) x),
$$

where we have noted $\psi_{l}(x)=\phi_{l+1}^{\text {odd }}(x)$ and $E_{l}=\mathcal{E}_{l+1}$. In particular, the fundamental and first excited eigenstates are $\psi_{1}^{(0)}(x)=\sin ^{2}(x)$ and $\psi_{2}^{(0)}(x)=\sin ^{2} x \cos x . V^{(0)}$ can also be considered as the image via $A\left(w_{0}\right)$ of the infinite square well

$$
V_{S W}(x)=\left\{\begin{array}{c}
0, \text { if } x \in] 0, \pi[ \\
+\infty, \text { if } x \notin] 0, \pi[
\end{array}\right.
$$

$V^{(0)}$ being a particular TDPT potential (10) with $n=0, m=1$, we can consider that $V_{S W}$ correspond to the limit case $V_{S W}(x)=V(x ; 0,0)=\lim _{\mu, \nu \rightarrow 0} V(x ; \mu, \nu)$.

As it is well known $\stackrel{9,17-19}{-19}$, the TDPT potentials $V(x ; \mu, \nu)$ are second category translationally shape invariant potentials (TSIP), the SUSY QM partner of $V(x ; \mu, \nu)$ being given by

$$
\widetilde{V}(x ; \mu, \nu)=V(x ; \mu+1, \nu+1),
$$

for $\mu, \nu>0$ and

$$
\begin{cases}\widetilde{V}(x ; \mu, 0)=V(x ; \mu+1,0), & \mu>0 \\ \widetilde{V}(x ; 0, \nu)=V(x ; 0, \nu+1), & \nu>0 .\end{cases}
$$

Starting from $V^{(0)}(x)=V(x ; 1,0)$, we can build a hierarchy of $p-1$ extensions of via successive SUSY QM partnerships, that is, via a chain of $p-1$ DBT based on the successive ground states. From Eq(32) and Eq(18), we deduce that the final potential is $V(x ; p, 0)$ and can be seen as the end of a chain of $p$ extensions which starts from the zero potential $V(x)$ and which is associated to the p-uple $N_{p}=(0, \ldots, p-1)$, ie to the set of seeds eigenfunctions

$\left(\psi_{0}(x), \ldots, \psi_{p-1}(x)\right)=(\sin (x), \ldots, \sin (p x))$. Using a straightforward recurrence, we deduce that the ground state of $V^{\left(N_{p}\right)}(x)=V(x ; p, 0)=p(p+1) / \sin ^{2} x$ at energy $E_{p}$ is

$$
\psi_{p}^{\left(N_{p}\right)}(x)=\sin ^{p+1} x .
$$

Since is even with respect to $\pi / 2$, its eigenfunctions $\psi_{n}^{\left(N_{p}\right)}$ are repectively symmetric (if $n-p$ is even) or antisymmetric (if $n-p$ is odd) with respect to this point. An immediate consequence is that its first excited eigenstate (at energy $\left.E_{p+1}\right) \psi_{p+1}^{\left(N_{p}\right)}$ has only one node on $] 0, \pi[$ which is always located at $x=\pi / 2$ independently of $p$. From Eq(33) we deduce

$$
\psi_{p+1}^{\left(N_{p}\right)}(x) \sim \widehat{A}^{+}\left(w_{p+1}^{\left(N_{p+1}\right)}\right) \psi_{p+1}^{\left(N_{p+1}\right)}(x) \sim \cos x \sin ^{p+1} x .
$$

Suppose that we continue the chain of extension and build the following DBT from this first excited eigenstate. The obtained potential is then singular and presents a pole of second order at $x=\pi / 2 . \psi_{p+1}^{\left(N_{p}\right)}$ being the image of $\psi_{p+1}(x)=\sin ((p+2) x)$ by the chain associated to $N_{p}$

$$
\psi_{p+1}^{\left(N_{p}\right)}(x)=\widehat{A}\left(w_{p}^{\left(N_{p-1}\right)}\right) \ldots \widehat{A}\left(w_{0}\right) \psi_{p+1}(x)
$$


the new chain is associated to the $(n+1)$-uple $\left(N_{p}, p+1\right)$ and the final extension is given by

$$
V^{\left(N_{p}, p+1\right)}(x)=\frac{2}{\cos ^{2} x}+\frac{(p+1)(p+2)}{\sin ^{2} x} .
$$

The presence of the additional "strong" singularity in $\pi / 2$ imposes a Dirichlet boundary condition at this point. In the spectrum of the restriction of $V^{\left(N_{p}, p+1\right)}$ to $] 0, \pi / 2$ [, half of the levels $E_{n}^{\left(N_{p}\right)}$ of the preceding potential $V^{\left(N_{p}\right)}$ are eliminated. These are all those associated to "even" (with respect to $\pi / 2$ ) eigenstates, that is to the even values of the quantum number $n-p$.

The spectrum of $V^{\left(N_{p}, p+1\right)}(x)$ contains then only the levels $E_{p+1+2(j+1)}, j \geq 0$, and the associated eigenstates $\psi_{p+1+2(j+1)}^{\left(N_{p}, p+1\right)}$ are the images of the initial eigenstates $\psi_{p+1+2(j+1)}$ via the chain of BDT corresponding to the $(p+1)-$ uple $\left(N_{p}, p+1\right)=(0, \ldots, p-1, p+1)$. Starting from $V^{\left(N_{p}, p+1\right)}$ we can continue the chain by using standard SUSY QM partnerships, all the extended potentials thus generated being perfectly regular on $] 0, \pi / 2\left[\right.$. But $V^{\left(N_{p}, p+1\right)}$ is also a TDPT

$$
V^{\left(N_{p}, p+1\right)}(x)=V(x ; p+1,1) .
$$

Consequently the shape invariance property $\mathrm{Eq}$ (31) implies that after $q$ steps, we obtain as final extension

$$
V^{\left(N_{p}, p+1, \ldots, p+q\right)}(x)=V(x ; p+q, q) .
$$

This shows in an explicite way what is exactly the chain of DBT which permit to build the general TDPT potential of integer parameters as a rational (in $\sin x$ ) extension of the free particle system.

The Crum formula gives then

$$
V(x ; p+q, q)=-2\left(\ln W\left(\psi_{0}, \ldots, \psi_{p-1}, \psi_{p+1,}, \ldots, \psi_{p-1+2 j}, \ldots, \psi_{p-1+2 q} \mid x\right)\right)^{\prime \prime},
$$

or, noting $l=p+q$,

$$
\begin{aligned}
V(x ; l, q) & =\frac{q(q+1)}{\cos ^{2} x}+\frac{l(l+1)}{\sin ^{2} x} \\
& =-2(\ln W(\sin x, \ldots, \sin ((l-q) x), \sin ((l-q+2) x), \ldots, \sin ((l-q+2 j) x), \ldots, \sin ((l+q) x) \mid x))^{\prime \prime},
\end{aligned}
$$

which is precisely the Gaillard-Matveev result.

\section{FROM THE CONSTANT POTENTIAL TO THE BESSEL POTENTIAL AND A WRONSKIAN RAYLEIGH FORMULA.}

To generate the TDPT potentials from the constant one, we start with a DBT built from any (physical) diffusion state of the constant potential. Nevertheless, we are not limited to this case and on a formal point of view we can use any eigenfunction, physical or not to build this DBT. In particular, we can choose as seed eigenfunction $\psi_{0}(x)=x=\lim _{k \rightarrow 0} \psi_{k}(x)$ whose associated eigenvalue $E_{0}=0$ is located at the lower boundary of the physical spectrum of $V$. The extended potential generated by the DBT $A\left(w_{0}\right)$ is

$$
V^{(0)}(x)=2 / x^{2}=V_{B}(x, 1),
$$

that is, the first Bessel potential of integer parameter, the general form of which being $V_{B}(x ; a)=a(a+1) / x^{2}$.

The two partner hamiltonians $\widehat{H}$ and $\widehat{H}^{(0)}$ are strictly isospectral but the levels of are no more degenerated since the strong singularity of $V^{(0)}$ at the origin imposes to retain in the spectrum of $\widehat{H}$ only the eigenstates which satisfy the Dirichlet boundary condition at the origin

$$
\psi\left(0^{+}\right)=0
$$


The physical spectrum of $\widehat{H}^{(0)}$ is then

$$
\left\{\begin{array}{c}
\left.E_{k}^{(0)}=k^{2} \in\right] 0,+\infty[, k>0 \\
\psi_{k}^{(0)}(x)=\frac{1}{E_{k}-E_{0}} \widehat{A}\left(w_{0}\right) \psi_{k}(x)=(-\sin (k x)+k x \cos (k x)) / k^{3} x
\end{array}\right.
$$

the unphysical "fundamental" eigenfunction for $k=0$ being $\psi_{0}^{(0)}(x)=x^{2} \sim \lim _{k \rightarrow 0} \psi_{k}^{(0)}(x)$.

We can start to build a chain of isospectral potentials by successive DBT based on the "fundamental" unphysical eigenfunctions of zero energy which are recessive at the origin (satisfying the Dirichlet boundary conditions at the origin). An immediate recurrence gives, for $N_{m}=\underbrace{(0, \ldots, 0)}_{m \text { im }}$

$$
\left\{\begin{array}{c}
\psi_{0}^{\left(N_{m}\right)}(x)=x^{m+1} \\
V^{\left(N_{m}\right)}(x)=V_{B}(x ; m)=m(m+1) / x^{2}
\end{array}\right.
$$

All the extensions $V^{\left(N_{m}\right)}$ subject to the Dirichlet boundary condition at the origin are then strictly isospectral to $V^{(0)}$ with a unique energy band $] 0,+\infty[$. These results are naturally well-known and this constitutes the first example of application that Darboux gave of his method in his first article on the subject 20 . They are also one of the key ingredient given by Duistermaat and Grünbaum ${ }^{21}$ in the proof that every trivial monodromy potential decaying at infinity can be considered as a rational extension of the free particle potential.

Nevertheless, the question of the Wronskian representation of the extended potentials Eq(44) and of their eigenfunctions is less trivial. Indeed, we are now in a case of confluency where all the successive DBT are built from eigenfunctions associated to the same energy. Then we cannot use the Crum formulas to express $V^{\left(N_{m}\right)}$ and its eigenstates $\psi_{k}^{\left(N_{m}\right)}$ in terms of Wronskians of distinct "seeds functions" (note that $\widehat{A}\left(w_{0}^{\left(N_{m}\right)}\right)$ is an annihilator for $\psi_{0}^{\left(N_{m}\right)}$ and that $\psi_{0}^{\left(N_{m+1}\right)}$ cannot be considered as the image of $\psi_{0}^{\left(N_{m}\right)}$ by the DBT $\left.A\left(w_{0}^{\left(N_{m}\right)}\right)\right)$. In this case, we can however use Matveev's formulas 22,23 and express $V^{\left(N_{m}\right)}$ and $\psi_{k}^{\left(N_{m}\right)}$ in terms of "generalized Wronskians":

Matveev's formulas: In the confluent case where the repeated DBT are built on eigenfunctions associated to the same value $k_{0}$ of the spectral parameter $k(N_{m}=\underbrace{\left(k_{0}, \ldots, k_{0}\right)}_{m \text { times }})$, we can write

$$
\left\{\begin{array}{c}
V^{\left(N_{m}\right)}(x)=V(x)-2\left(\ln W\left(\psi_{\kappa},\left.\frac{\partial^{i 1} \psi_{\kappa}}{\partial \kappa^{i} 1}\right|_{\kappa=k_{0}}, \ldots,\left.\frac{\partial^{i} m-1}{\partial \kappa^{m-1}}\right|_{\kappa=k_{0}} \mid x\right)\right)^{\prime \prime} \\
\psi_{k}^{\left(N_{m}\right)}(x)=\frac{W\left(\psi_{k_{0}},\left.\frac{\partial^{i} 1 \psi_{\kappa}}{\partial \kappa^{i} 1}\right|_{\kappa=k_{0}}, \ldots,\left.\frac{\partial^{i} m-1}{\partial \kappa^{m-1}}\right|_{\kappa=k_{0}}, \psi_{k} \mid x\right)}{W\left(\psi_{k_{0}},\left.\frac{\partial^{i} 1 \psi_{\kappa}}{\partial \kappa^{i} 1}\right|_{\kappa=k_{0}}, \ldots,\left.\frac{\partial^{i} m-1}{\partial \kappa^{m-1}}\right|_{\kappa=k_{0}} \mid x\right)}
\end{array}\right.
$$

where the $\left.\frac{\partial^{i} j \psi_{\kappa}}{\partial \kappa^{i} j}\right|_{\kappa=k_{0}}, j=0, \ldots, m-1$, are the $m$ first non-zero derivative of $\psi_{\kappa}$ with respect to the spectral parameter $\kappa$ at the value $\kappa=k_{0}$. In our case, for $k_{0}=0$, we have

$$
\psi_{k}(x)=\sum_{n=0}^{\infty}(-1)^{n} \frac{x^{2 n+1}}{(2 n+1) !}(k)^{2 n}
$$

The sequence of the $i_{j}$ is given by $i_{j}=2 j, j \geq 0$ with

$$
\left(\frac{\partial^{2 j} \psi_{\kappa}(x)}{\partial \kappa^{2 j}}\right)_{\kappa=0}=(-1)^{j} \frac{x^{2 j+1}}{2 j+1}
$$

and the Matveev formula for the potential gives

$$
V(x ; m)=2\left(\ln W\left(x, x^{3}, \ldots, x^{2 m-1} \mid x\right)\right)^{\prime \prime}
$$


Darboux's result is readily recovered by using standard properties of the Wronskians 26 which allow us to write

$$
W\left(x, x^{3}, \ldots, x^{2 m-1} \mid x\right)=\left.x^{m}\left(\frac{d y}{d x}\right)^{m(m-1) / 2} W\left(1, y, \ldots, y^{m-1} \mid y\right)\right|_{y=x^{2}}=x^{m(m+1) / 2} 2^{m(m-1) / 2} \prod_{j=1}^{m-1} j !
$$

that is, $V^{\left(N_{m}\right)}(x)=V_{B}(x ; m)=m(m+1) / x^{2}$.

More interesting is the case of the eigenfunctions. Since $\psi_{k}^{\left(N_{m}\right)}$ is a solution of

$$
\psi^{\prime \prime}(x)+\left(k^{2}-\frac{m(m+1)}{x^{2}}\right) \psi(x)=0
$$

satisfying the Dirichlet boundary condition at the origin, it can then be written as

$$
\psi_{k}^{\left(N_{m}\right)}(x) \sim x^{1 / 2} \mathbf{J}_{m+1 / 2}(k x)=\sqrt{\frac{2}{\pi}} x \mathbf{j}_{m}(k x),
$$

where $\mathbf{J}_{m+1 / 2}(x)$ and $\mathbf{j}_{m}(x)$ are the usual Bessel and spherical Bessel functions 24,25 .

But from the second Matveev's formula we also have

$$
\psi_{k}^{\left(N_{m}\right)}(x)=\frac{W\left(x, x^{3}, \ldots, x^{2 m-1}, \sin (k x) / k \mid x\right)}{W\left(x, x^{3}, \ldots, x^{2 m-1} \mid x\right)} .
$$

This can be considered as a Wronskian version of the Rayleigh formula for Bessel functions 24 . Indeed, the same handling as before ${ }^{26}$ applied to the Wronskian at the numerator of Eq(152) gives

$$
W\left(x, x^{3}, \ldots, x^{2 m-1}, \sin (k x) / k \mid x\right)=2^{m(m+1) / 2} x^{(m+1)(m+2) / 2} W\left(1, y, \ldots, y^{m-1}, g(y) \mid y\right),
$$

where $y=x^{2}$ and $g(y)=\operatorname{sinc}(k x)=\sin (k x) / k x$. Then

$$
W\left(x, x^{3}, \ldots, x^{2 m-1}, \sin (k x) / k \mid x\right)=2^{m(m+1) / 2} x^{(m+1)(m+2) / 2}\left(\prod_{j=1}^{m-1} j !\right) g^{(m)}(y)
$$

and with Eq(49), we finally obtain

$$
\psi_{k}^{\left(N_{m}\right)}(x)=\frac{x^{m+1}}{2^{m}}\left(\frac{1}{x} \frac{d}{d x}\right)^{m} \sin \mathrm{c}(k x) .
$$

\section{CONCLUSION}

In this letter we have shown how to recover in a simple way the content of the Gaillard-Matveev theorem which provides a Wronskian representation for the TDPT potentials. This is directly achieved by combining the Crum formula and shape invariance arguments applied to specific singular extensions of the constant potential. The same reasoning can be adapted to obtain Wronskian representations for the eigenfunctions of the Bessel potentials. This confluent case necessitates to employ Matveev's generalized Wronskians, obtaining then a Wronskian version of the Rayleigh formula.

$\mathrm{In}^{3}$, Gaillard and Matveev also consider the case of the discrete Darboux-Pöschl-Teller potentials (DDPT), for which they give Casoratian representation formulas. A treatment of these discrete systems by the preceding approach will be the object of further investigations. 


\section{ACKNOWLEDGEMENTS}

I would like to thank R. Milson and D. Gomez-Ullate for stimulating discussions.

${ }^{1}$ G. Darboux, Leçons sur la théorie générale des surfaces et les applications géométriques du calcul infinitésimal, vol 2, 2nd edition (Gauthier-Villars, Paris, 1915).

2 P. Gaillard and V. B. Matveev, "Wronskian addition formula and its application", vol. MPI 02-31 (Bonn: Max-PlanckInstitut für Mathematik) 1-17 (2002).

3 P. Gaillard and V. B. Matveev, "Wronskian and Casorati determinant representations for Darboux-Pöschl-Teller potentials and their difference extensions", J. Phys. A: Math. Gen. 42, 404009 (2009).

4 J. Casahorran, "A family of supersymmetric quantum mechanics models with singular superpotentials", Phys. Lett. B 156, 425-428 (1991).

5 P. K. Panigrahi and U. P. Sukhatme, "Singular superpotentials in supersymmetric quantum mechanics", Phys. Lett. A 178, 251-257 (1993).

6 M. Robnik, "Supersymmetric quantum mechanics based on higher excited states", J. Phys. A 30, 1287-1294 (1997).

7 I. F. Marquez, J. Negro and L.M. Nieto, "Factorization method and singular hamiltonians", J. Phys. A: Math. Gen. 31, 4115-4125 (1998).

8 I. Marquette, "Singular isotonic oscillator, supersymmetry and superintegrability", SIGMA 8, 063 (2012).

9 Y. Grandati and A. Bérard, "Rational solutions for the Riccati-Schrödinger equations associated to translationally shape invariant potentials", Ann. Phys. 325, 1235-1259 (2010).

10 J. F. Cariñena and A. Ramos, "Integrability of Riccati equation from a group theoretical viewpoint", Int. J. Mod. Phys. A 14, 1935-1951 (1999).

11 J. F. Cariñena, A. Ramos and D. J. Fernandez, "Group theoretical approach to the intertwined hamiltonians", Ann. Phys. 292, 42-66 (2001).

12 C. V. Sukumar, "Supersymmetry, factorization of the Schrödinger equation and an hamiltonian hierarchy", J. Phys. A 18, L57-L61 (1985).

13 M. M. Crum, "Associated Sturm-Liouville systems", Q. J. Math. 6, 121-127 (1955).

14 V. B. Matveev and M. A. Salle, Darboux Transformations and Solitons (Springer-Verlag, Berlin, 1991).

15 W. Frank, D. Land and R. Spector, "Singular potentials", Rev. Mod. Phys. 43, 36-98 (1971).

16 L. Lathouwers, "The hamiltonian $H=\left(-\frac{1}{2}\right) \frac{d^{2}}{d x^{2}}+\frac{x^{2}}{2}+\frac{\lambda}{x^{2}}$ reobserved", J. Math. Phys. 16, 1393-1395 (1975).

17 F. Cooper, A. Khare and U. Sukhatme, Supersymmetry in Quantum Mechanics (World Scientific, Singapore, 2001).

18 R. Dutt, A. Khare and U. P. Sukhatme, "Supersymmetry, shape invariance and exactly solvable potentials", Am. J. Phys. 56, 163-168 (1988).

19 L. Gendenshtein, "Derivation of exact spectra of the Schrodinger equation by means of supersymmetry", JETP Lett. 38, 356-359 (1983).

${ }^{20}$ G. Darboux, "Sur une proposition relative aux équations linéaires", Comptes Rendus Acad.Sci. 94, 1456-1459 (1882).

21 J. J. Duistermaat and F. A. Grünbaum, "Differential equations in the spectral parameter", Comm. Math. Phys. 103, 177-240 (1986).

${ }^{22}$ V. B. Matveev, "Generalized Wronskian formula for solutions of the KdV equations: first applications", Phys. Lett. A 166, 205-208 (1992).

23 V. B. Matveev, "Positons: slowly decreasing analogues of solitons", Theor. Math. Phys. 131, $483-497$ (2002).

24 M. Abramowitz and I. A. Stegun, Hanbook of mathematical functions (Dover, New-York, 1972).

25 A. Erdélyi, W. Magnus, F. Oberhettinger and F. G. Tricomi, Higher transcendental functions (Mc Graw-Hill, New York, 1953).

26 T. Muir (revised and enlarged by W.H. Metzler), A treatise on the theory of determinants (Dover, New York, 1960). 